\title{
An Innovative Bridge Inspection Technique through Drive-by Vehicles - A Special Application on Prestress Loss Identification
}

\author{
Dr. Mijia Yang \\ University of North Dakota, USA
}

Prestress force loss in prestressed concrete bridge is inevitable; however it is the amount of the prestress force loss matters, which determines the safety of load-carrying members. More than $60 \%$ of all new and replaced bridges built in US are prestressed concrete bridges since the year 2000, which demands a fast and accurate inspection technique to monitor their prestress loss.

The existed direct and indirect methods used for prestress loss identification are all based on the measurement collected from sensors deployed on the outside or inside of the bridge, which is not only costly but also inconvenient. The current study proposes to detect the prestress force loss of the bridge through the analysis of vehicle responses, in which the principle of virtual works was adopted to take the effect of prestress into consideration. Through simulations, it is found that vehicle vertical acceleration shows a strong correlation with the prestress loss and light, low-frequency vehicles moving at low speeds have a better performance in detecting the bridge prestress loss than the heavy, high-frequency vehicles with high speeds. The advantage of the proposed method is that it only needs a few sensors installed on the vehicle, and works without interrupting the ongoing traffic. Based on the validated modeling results, the suggested technique provides an efficient and cost-effective tool for state agencies to maintain a large amount of prestressed bridges. 